
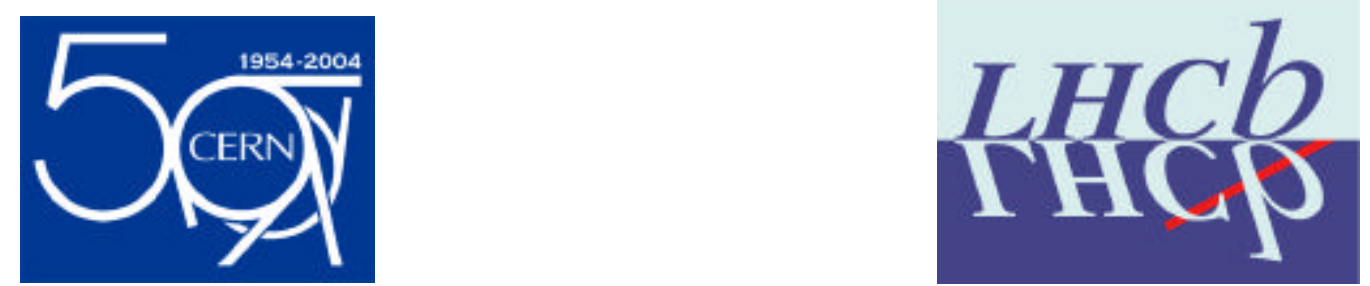

\title{
Performance studies of Pixel Hybrid Photon Detectors for the LHCb RICH counters
}

\author{
Gianluca Aglieri Rinella
}

CERN - European Organization for Nuclear Research DIEPA - University of Palermo, Italy

On behalf of the LHCb Collaboration - Pixel HPD group

Siena, May $25^{\text {th }} 2004$ 


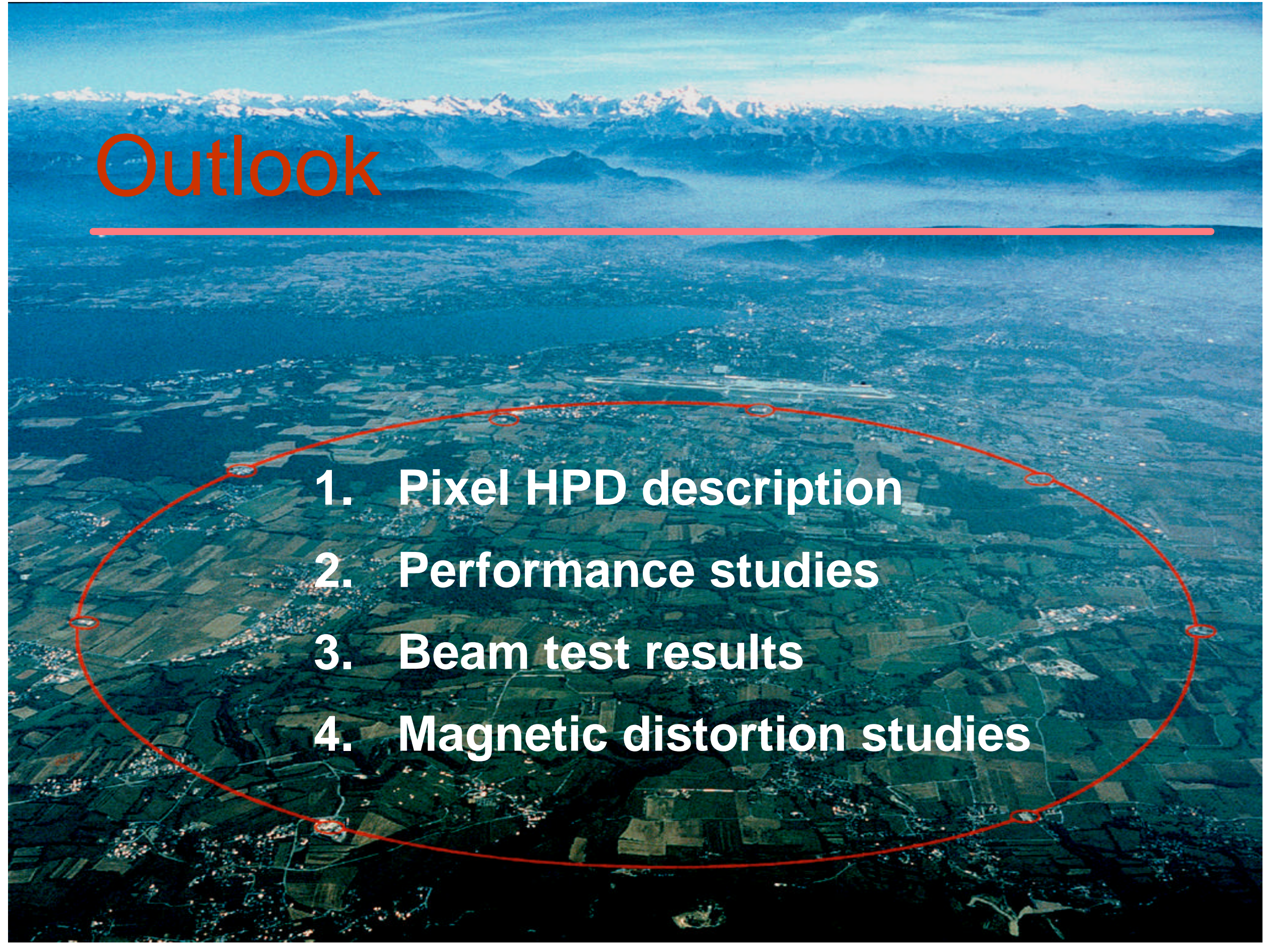




\section{The Pixel Hybrid Photon Detector}

The photon detectors of the LHCb - RICHs

- Particle identification at LHCb experiment: kaon identification by Ring Imaging Cherenkov counters over $1-100 \mathrm{GeV} / \mathrm{c}$ momentum range

Photon detectors requirements:

- Efficient single photon detection in the 200$600 \mathrm{~nm}$ wavelength range

- Large area coverage: $\mathbf{2 . 8} \mathrm{m}^{2}$

- Active/Total area ratio $>70 \%$

- $2.5 \times 2.5 \mathrm{~mm}^{2}$ granularity

- Time resolution: 25 ns

- Operation in fringe magnetic field of $\sim 25 \mathrm{G}$

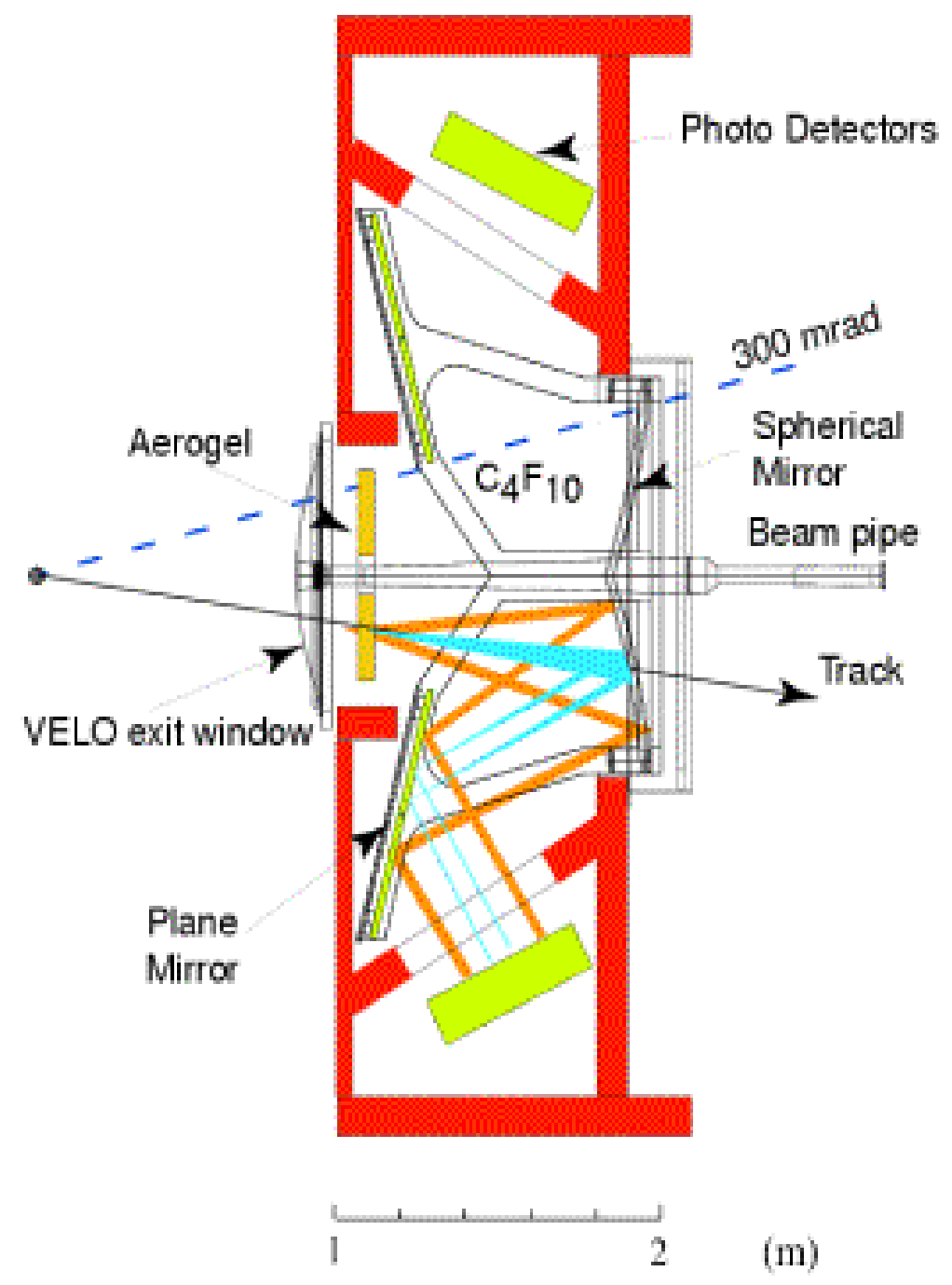




\section{Pixel HPD}

- Vacuum tube, diameter $80 \mathrm{~mm}$, height $\sim 120 \mathrm{~mm}$

- S20 multi-alkali photocathode on inner surface of the entrance quartz window, 22-27\% QE

- Cross-focusing electron optics ( 20kV, 5000 e-h pairs in the silicon), demagnification factor of 5

-Photo-electrons focused on the anode assembly $\left(16 \times 16 \mathrm{~mm}^{2}\right)$, fully encapsulated in the vacuum enclosure

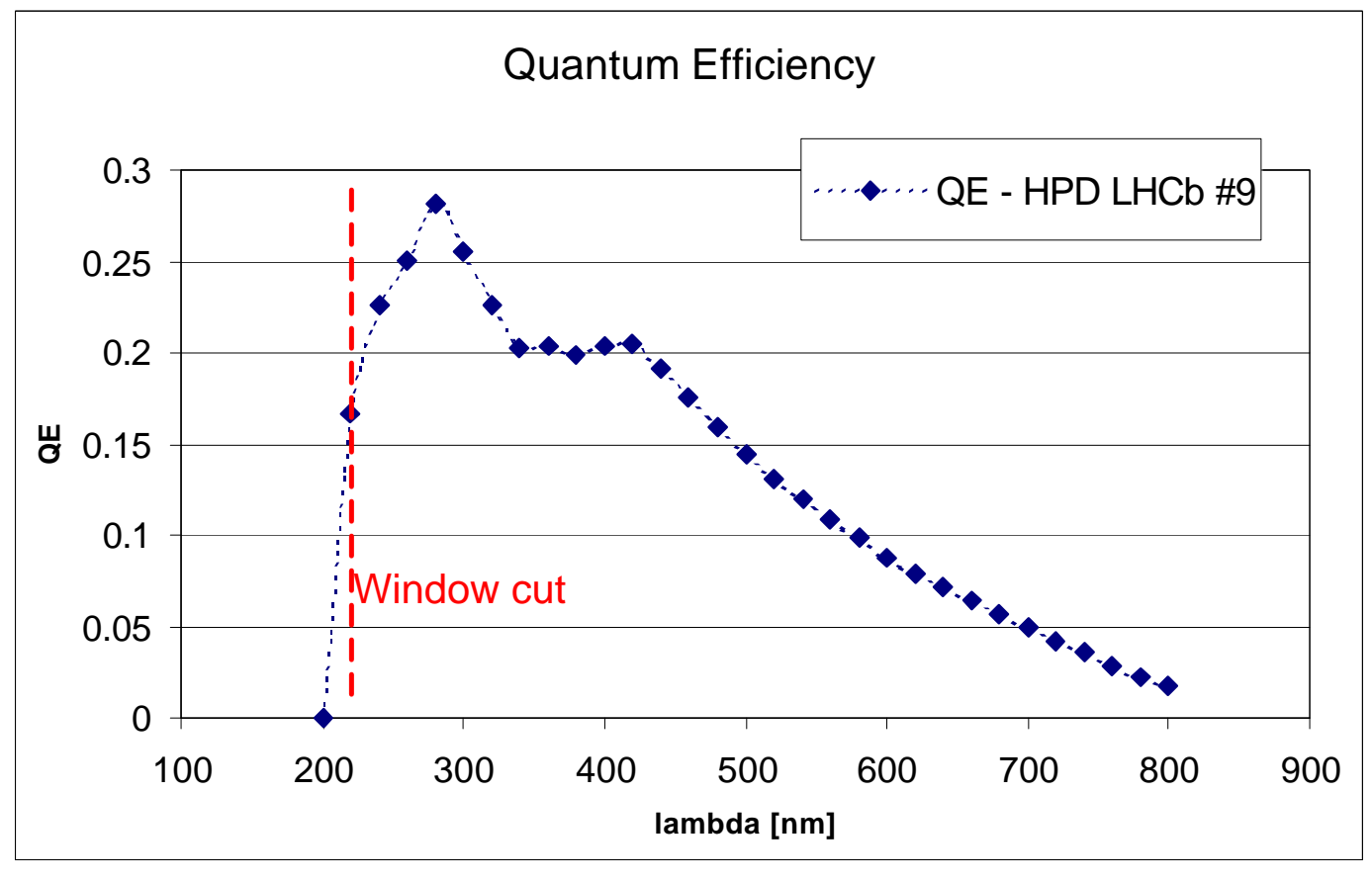

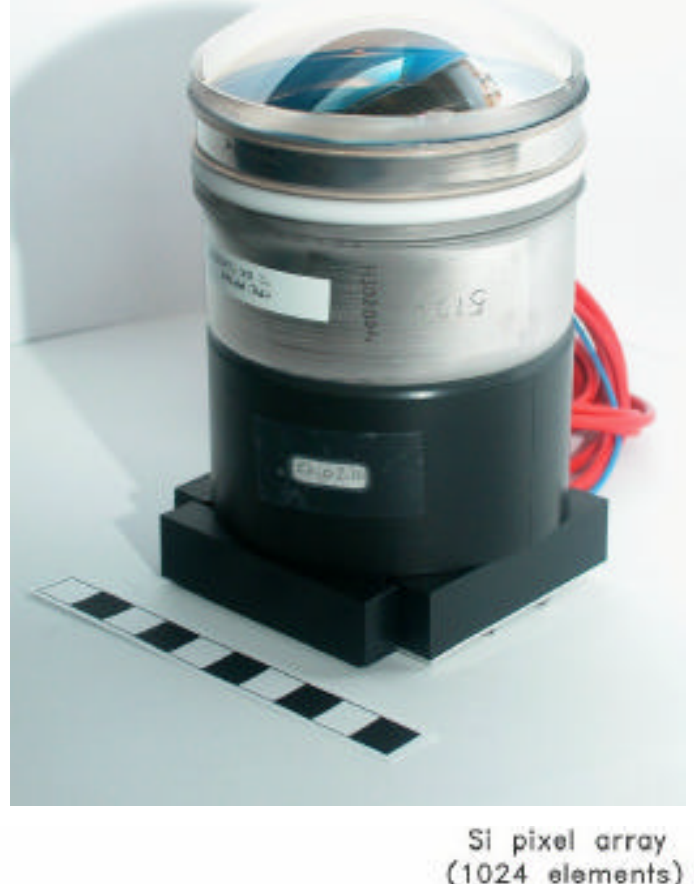

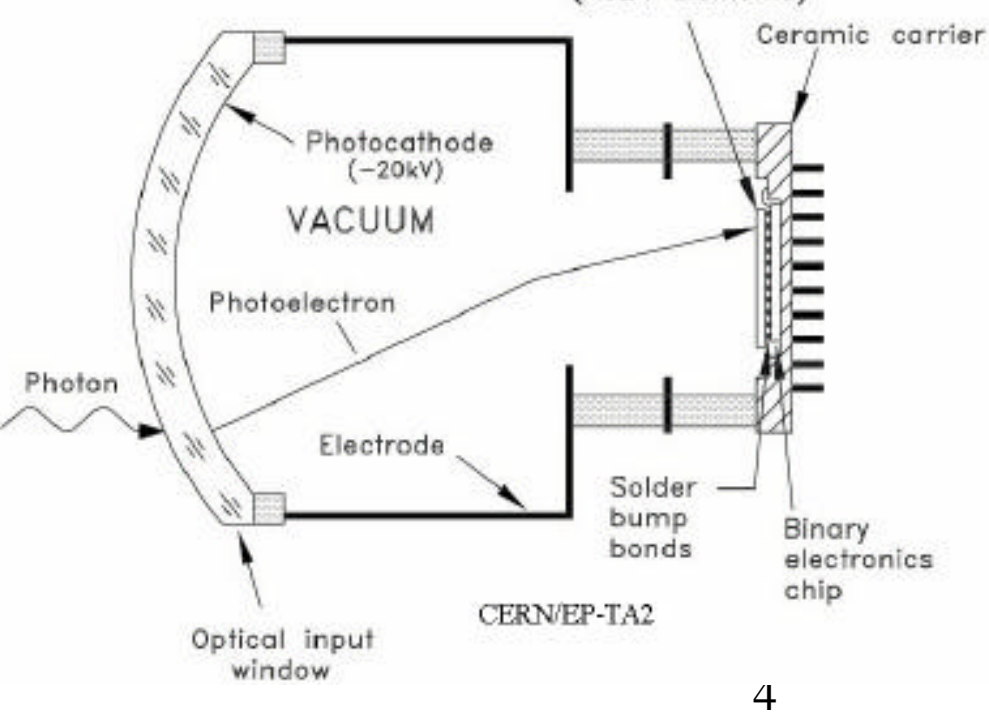




\section{Pixel HPD Anode}
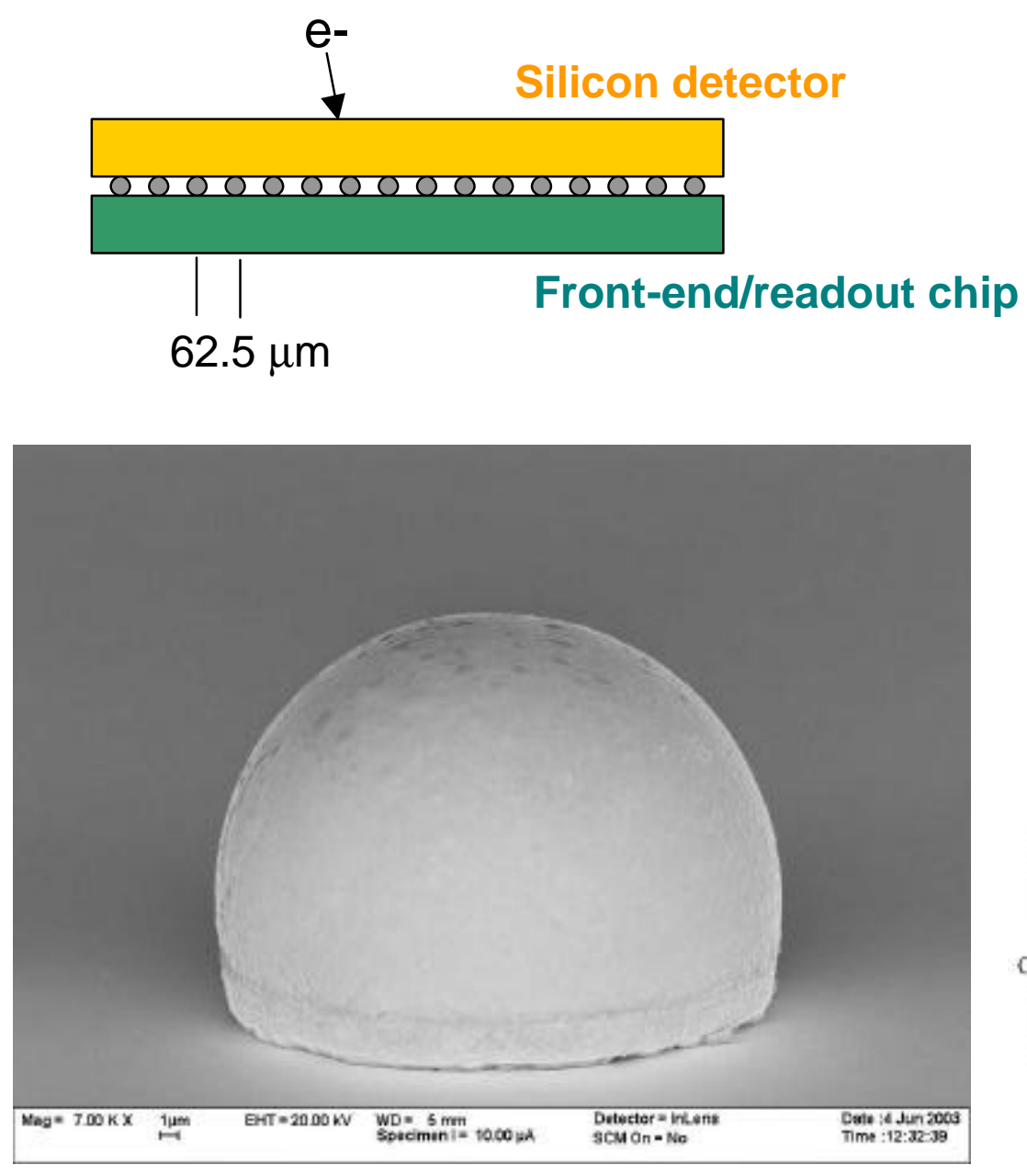

-Assembly: silicon detector, 32x256 matrix of $500 \times 62.5 \mu \mathrm{m}^{2}$ pixels bump-bonded onto the LHCbPIX1 CMOS readout chip

-8192 channels with charge pre-amp, 25ns peaking time, adjustable threshold discriminator, coincidence logic, memory, operating at $40 \mathrm{MHz}$ clock

-Digital OR grouping of 8 pixels for the $\mathrm{LHCb}$ experiment gives $1024=32 \times 32$ sensitive areas of $2.5 \times 2.5 \mathrm{~mm}^{2}$ on the entrance window, fully read-out in 800 ns through 32 parallel lines

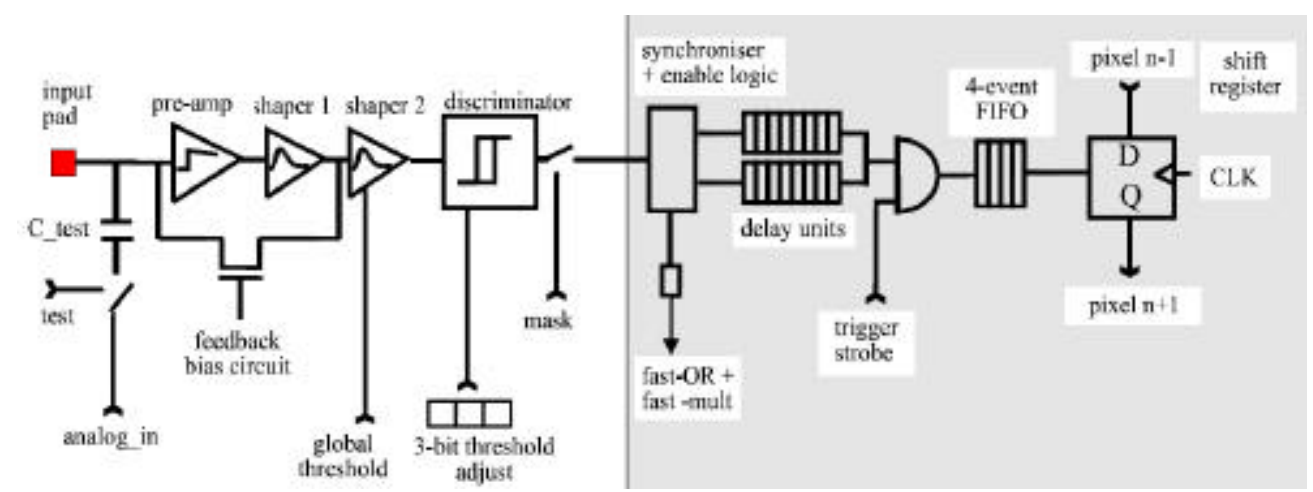




\section{Tube H.V. scan and Detector Bias scan}
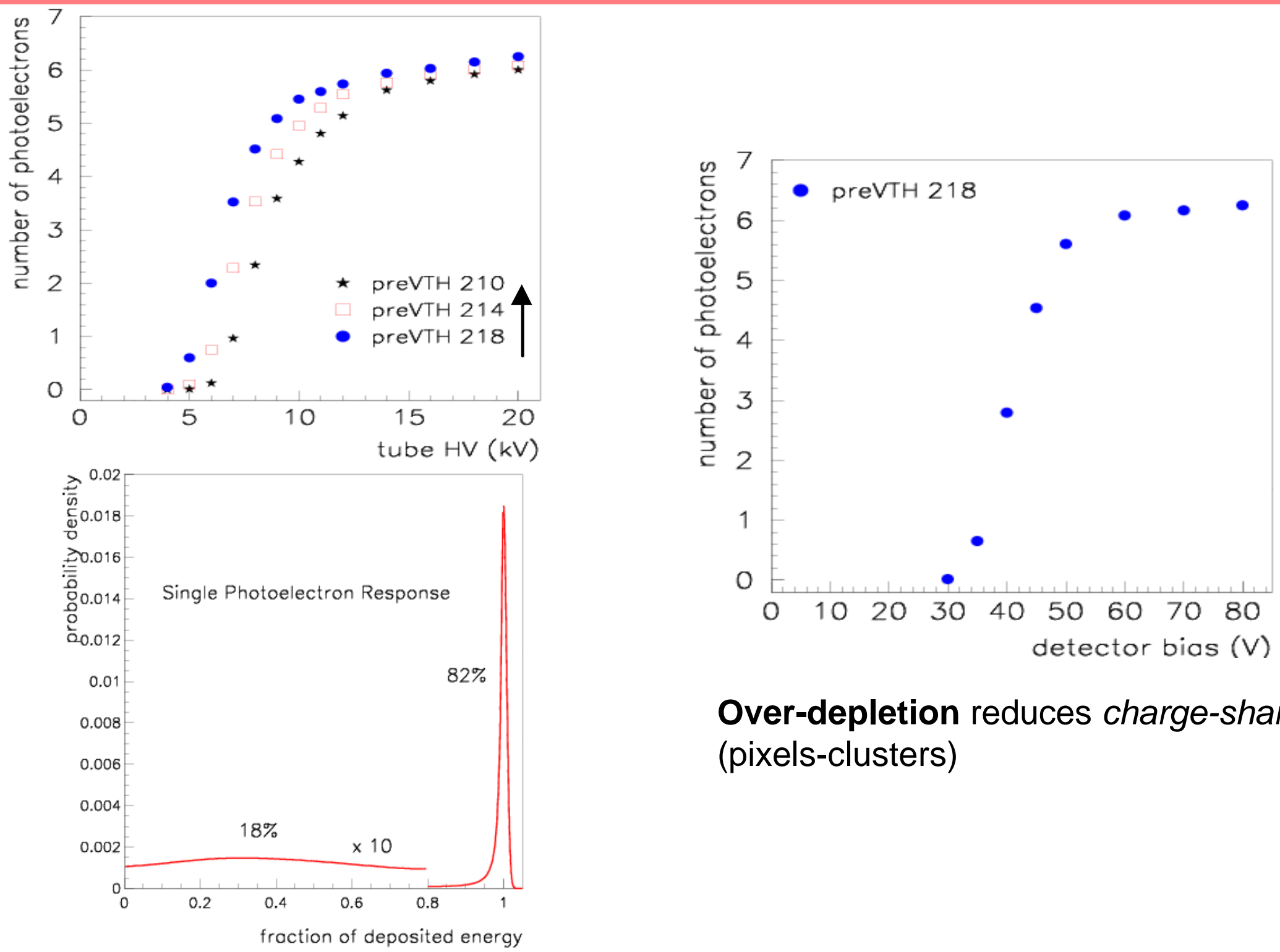

Over-depletion reduces charge-sharing (pixels-clusters) 


\section{Detection Efficiency}

Detection Efficiency $=\frac{\text { Number of clusters }>\text { Threshold }}{\text { Number of p.e. }}$

- Sources of losses: back-scattering ( 18\%), chargesharing, threshold

- Silicon detector total current analog signal (backpulse) sampled during run

- A fit of its spectrum allows for quantification of average number of incident p.e

- Binary data analysis considered pixel clustering

- Detection Efficiency: 88\% (@20 kV)

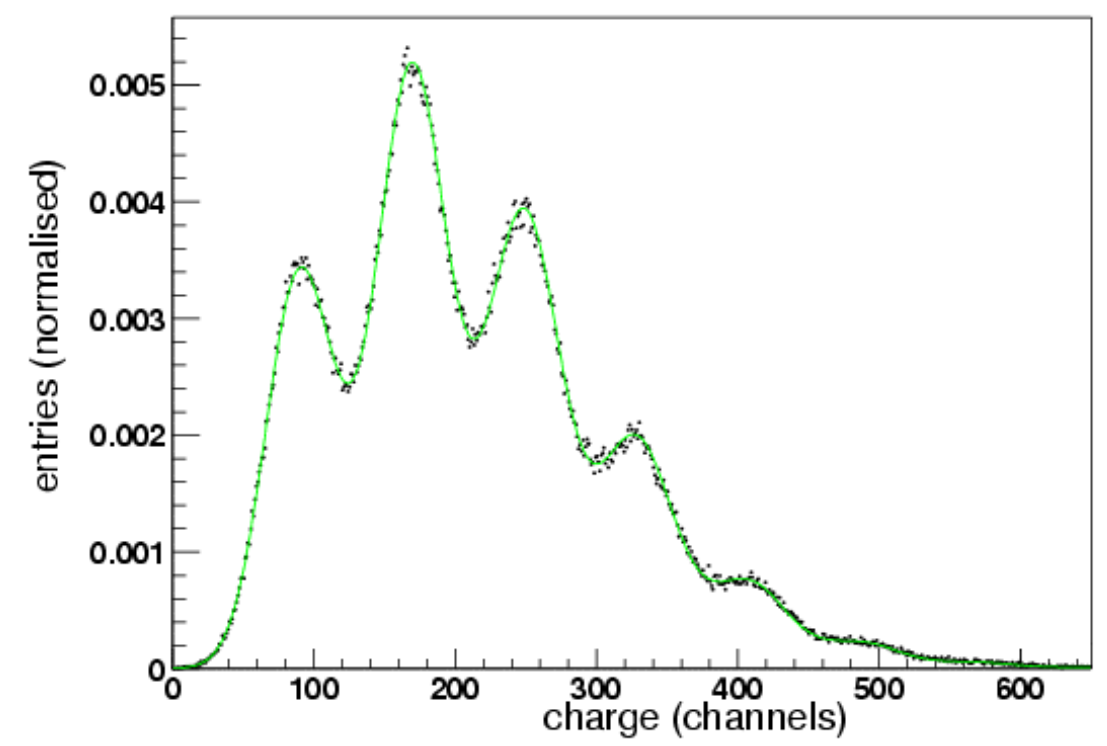

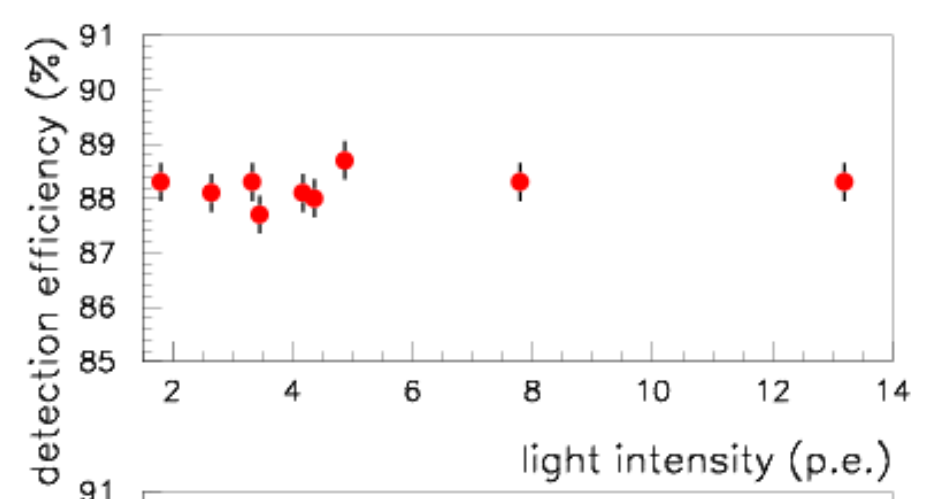
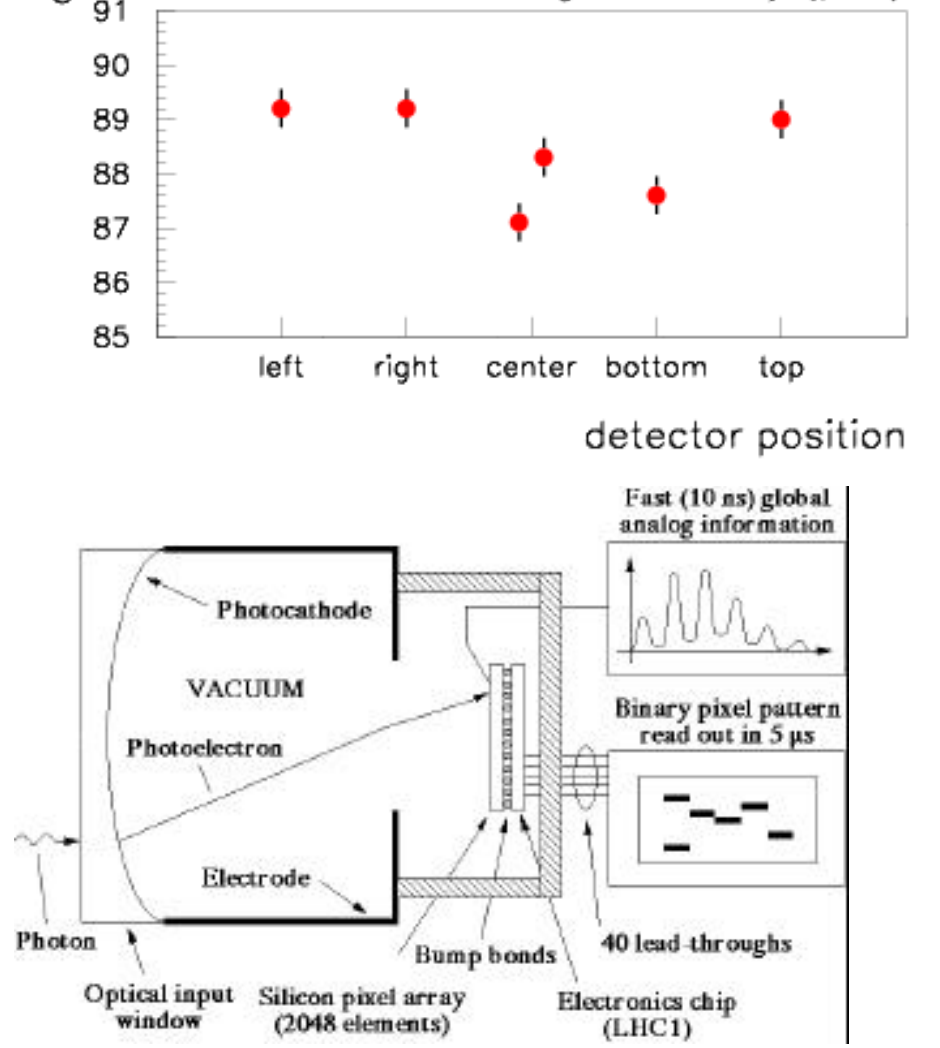
window (2048 elements) 


\section{Background}

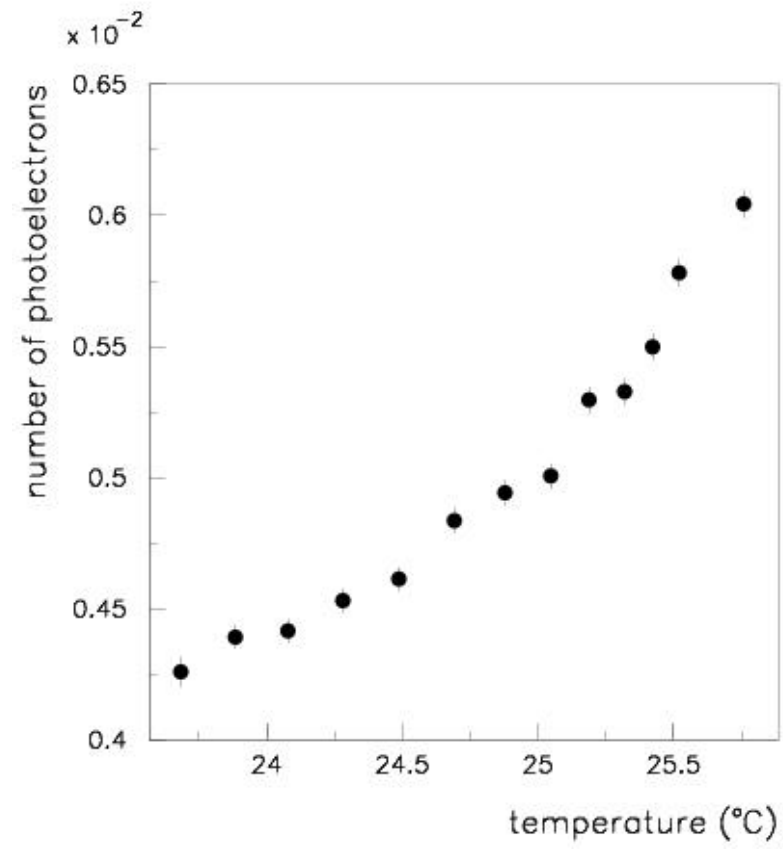

1. Dark counts

- Thermo-ionic emission from the photocathode is a source of background

- Typical dark-counts rate in the $40 \mathrm{MHz}$ prototypes of

$\sim 1 \mathrm{KHz} / \mathrm{cm}^{2}$

2. Ion feedback

- Ionisation of residual gas molecules and acceleration of ions towards cathode, where they cause emission of electrons

- Fingerprints: time delayed with respect to original signal hit of $\sim 250$ ns and large clusters

- $\quad$ Probability of ion feedback: $0.5 \%$ per primary photoelectron

- Well known in PMT: afterpulses 


\section{Test beams - HPD performance}

Prototype $40 \mathrm{MHz}$ HPDs were tested and used in two beam tests at CERN, August 2003 and October 2003 in Cherenkov imaging vessels, with air, N2 and Aerogel as radiators

Cherenkov rings from $10 \mathrm{GeV} / \mathrm{c}$ pions and electrons were recorded Measured Cherenkov angles: $20.6 \pm 1.3 \mathrm{mrad}$ and $25.6 \pm 1.5$ mrad

H.V. scan, detection efficiency measurements in agreement with laboratory measurements

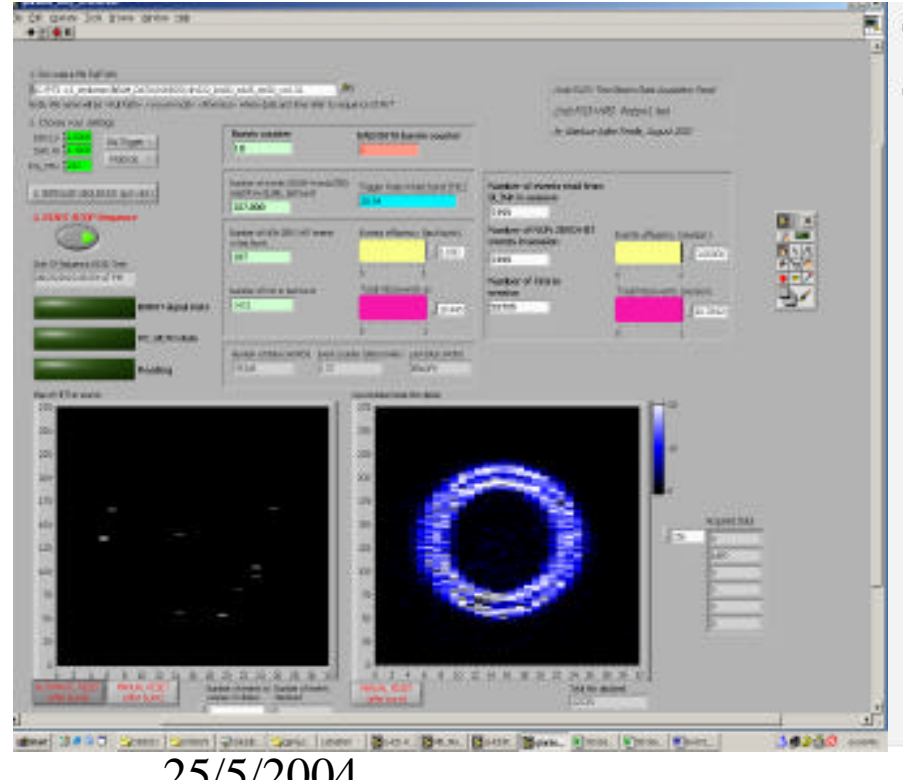

$25 / 5 / 2004$

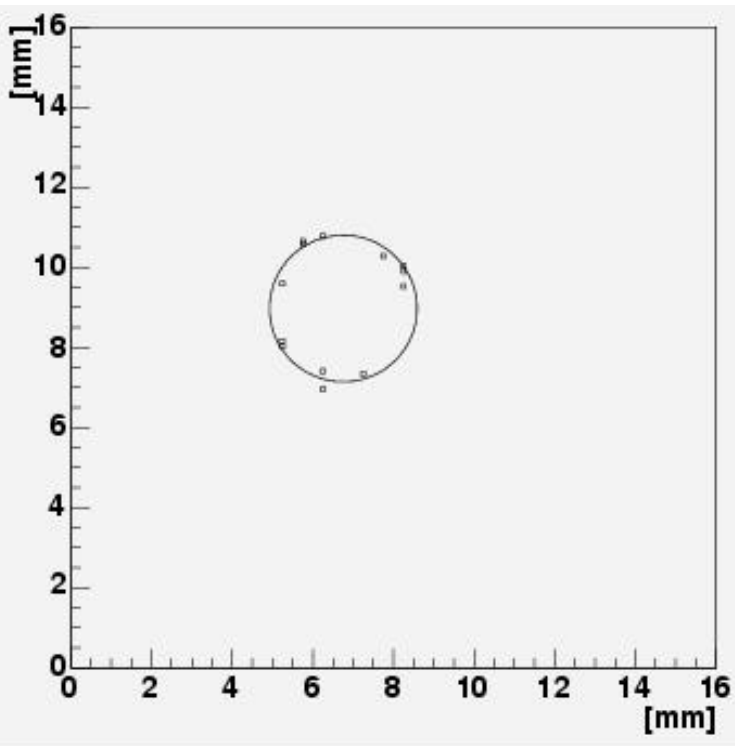

Gianluca Aglieri Rinella

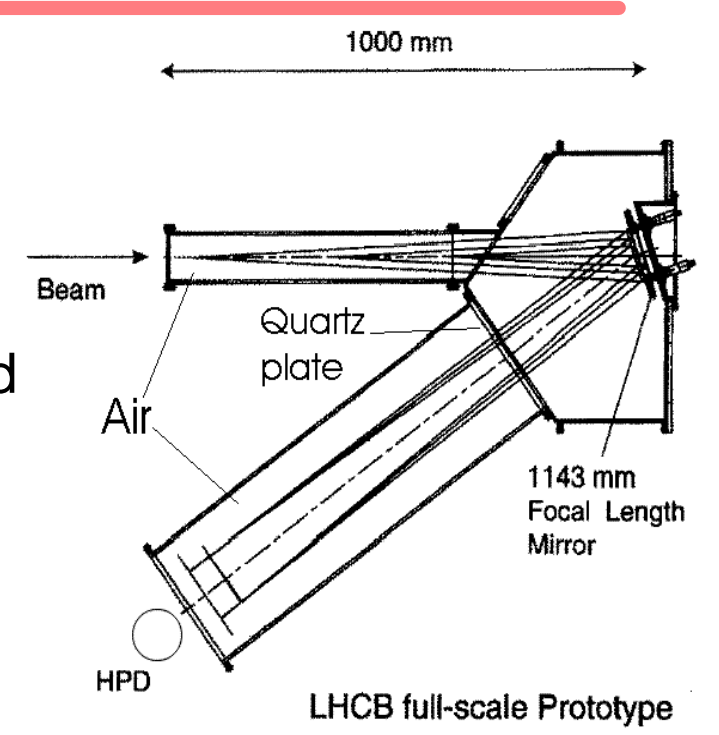

Radii distribution

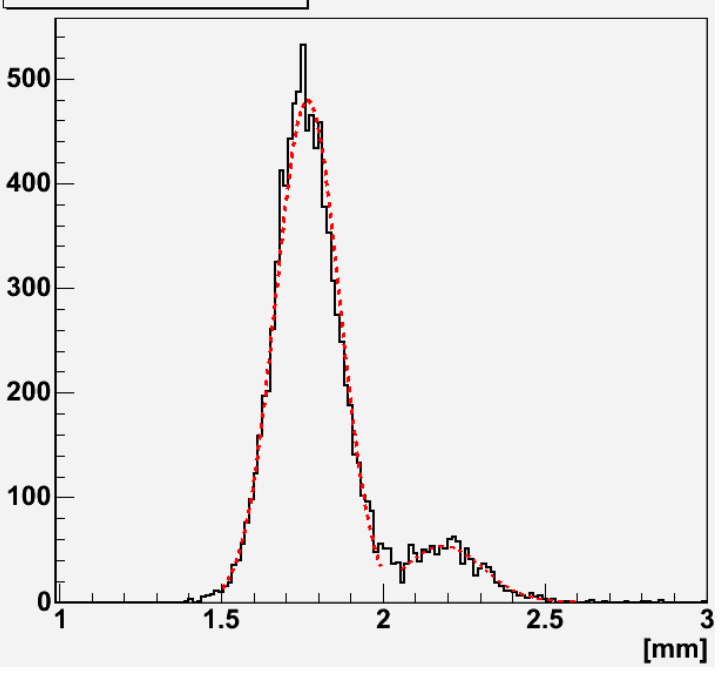

9 


\section{Test beams - HPD performance}

Radiator (N2) refractive index curve, losses for reflections at interfaces, HPD cathode Q.E., are used for average photon yield calculation

Correction for multiple hits probability is applied

The result is to be compared with the measured average cluster number to give an independent measurement of the Detection Efficiency

$$
N=\int_{\lambda}^{2 \pi Z^{2} \alpha L} \frac{1}{\lambda^{2}}\left[1-\frac{1}{n^{2}(\lambda) \cdot \beta^{2}}\right] \cdot R_{M}(\lambda) \cdot T(\lambda) \cdot Q E(\lambda) d \lambda
$$

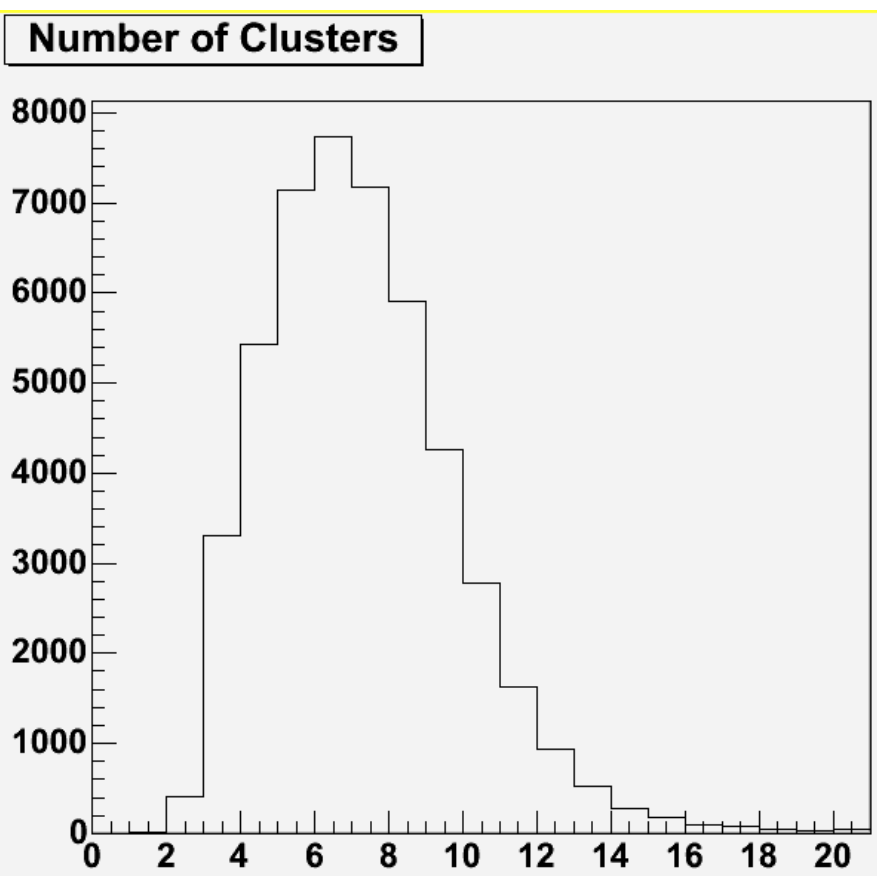

\begin{tabular}{|c|c|c|c|}
\hline Tube & $\begin{array}{c}\text { Expected } \\
\text { number of p.e. } \\
(100 \% \text { DE })\end{array}$ & $\begin{array}{c}\text { Measured } \\
\text { average of } \\
\text { p.e. }\end{array}$ & $\begin{array}{c}\text { Detection } \\
\text { Efficiency [\%] }\end{array}$ \\
\hline LHCb \#8 & $11.6( \pm 0.7)$ & 10.1 & $87.1 \pm 5.3$ \\
\hline LHCb \#9 & $12.8( \pm 0.8)$ & 10.6 & $82.8 \pm 5.2$ \\
\hline
\end{tabular}




\section{Magnetic distortions studies}

The HPDs have to be placed in the fringe field of the LHCb dipole magnet

Deviations of the electrons from the designed electrostatic trajectories because of the magnetic field, result in distortions of the image of the cathode

A large magnetic shielding box surrounds the photon detectors: residual magnetic field $\sim 25 \mathrm{G}$

HPDs could need individual magnetic shielding: cylindrical envelope of high permeability alloy for each tube

Residual distortions to be off line compensated restoring the excellent space resolution
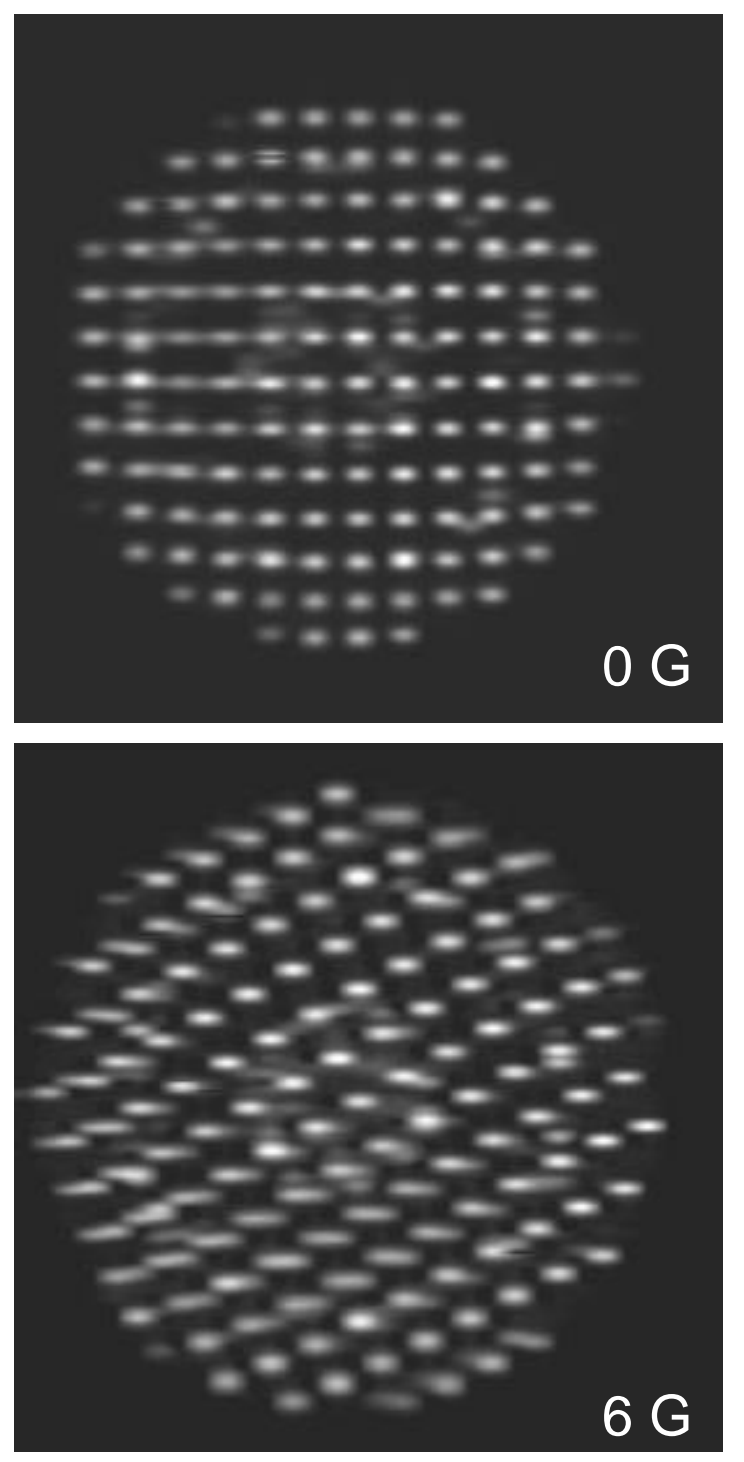


\section{Magnetic distortions studies}

Distortions in a shielded tube in a transverse field of $50 \mathrm{G}$ equivalent to bare tube in $2.5 \mathrm{G}$

Distortions in a shielded tube in an axial field of $50 \mathrm{G}$ equivalent to bare tube in $15 \mathrm{G}$

NO coverage losses with shielded tube up to $50 \mathrm{G}$

Parameterization of distortions

Distortion compensation algorithms (un-warping) to be developed

Detector calibration with a projected test pattern
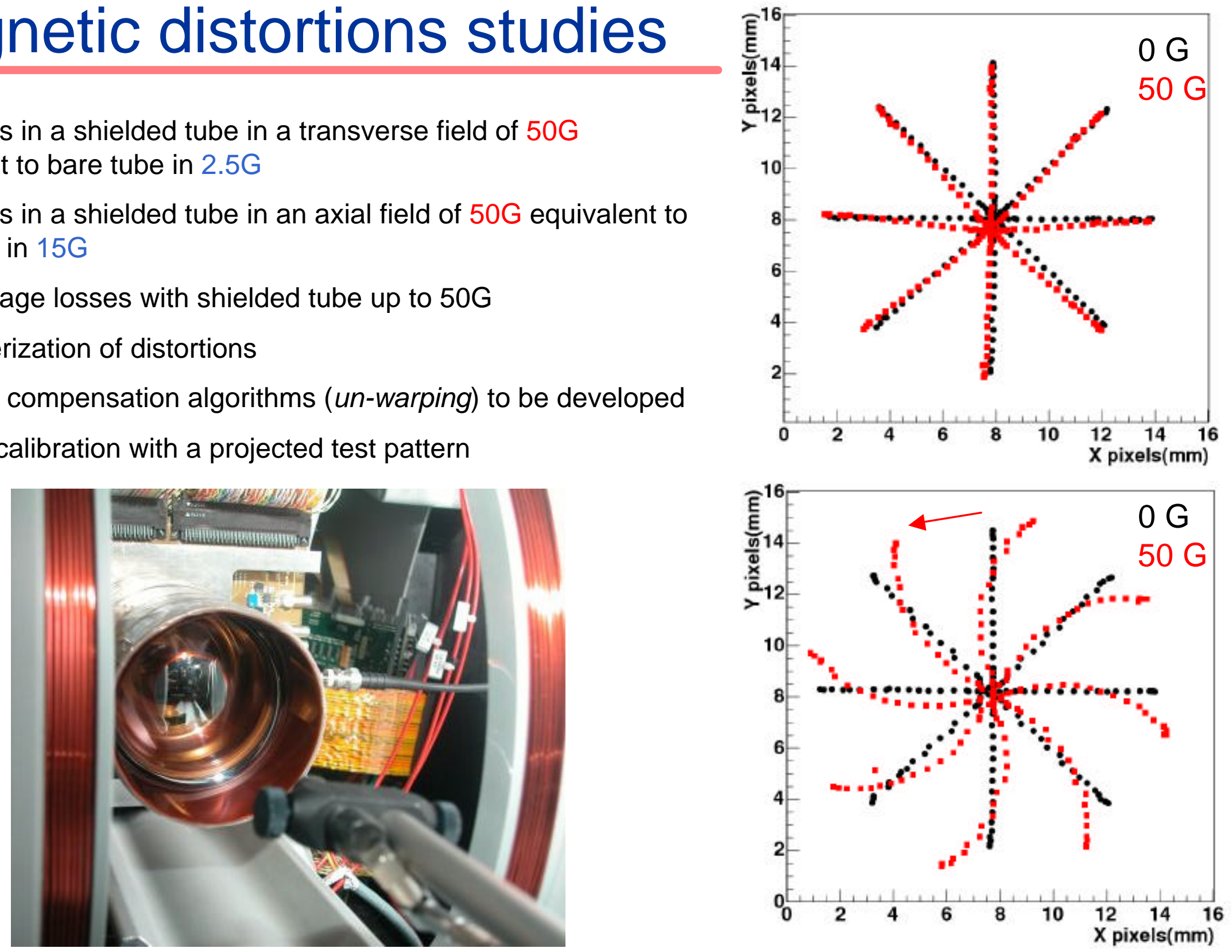


\section{Summary}

- Pixel Hybrid Photon detectors: single photon sensitive, large active area, fast, imaging detectors developed for the $\mathrm{LHCb} \mathrm{RICH}$ detectors

- Prototypes performance determined in laboratory measurements

- Final 6 prototypes fulfill experiment requirements

- HPDs used in two beam tests at CERN in a $\mathrm{RICH}$ vessel

- Detection efficiency $88 \%$

- Magnetic distortion compensation

- Production phase entered with 550 tubes to be produced 


\section{Spares}

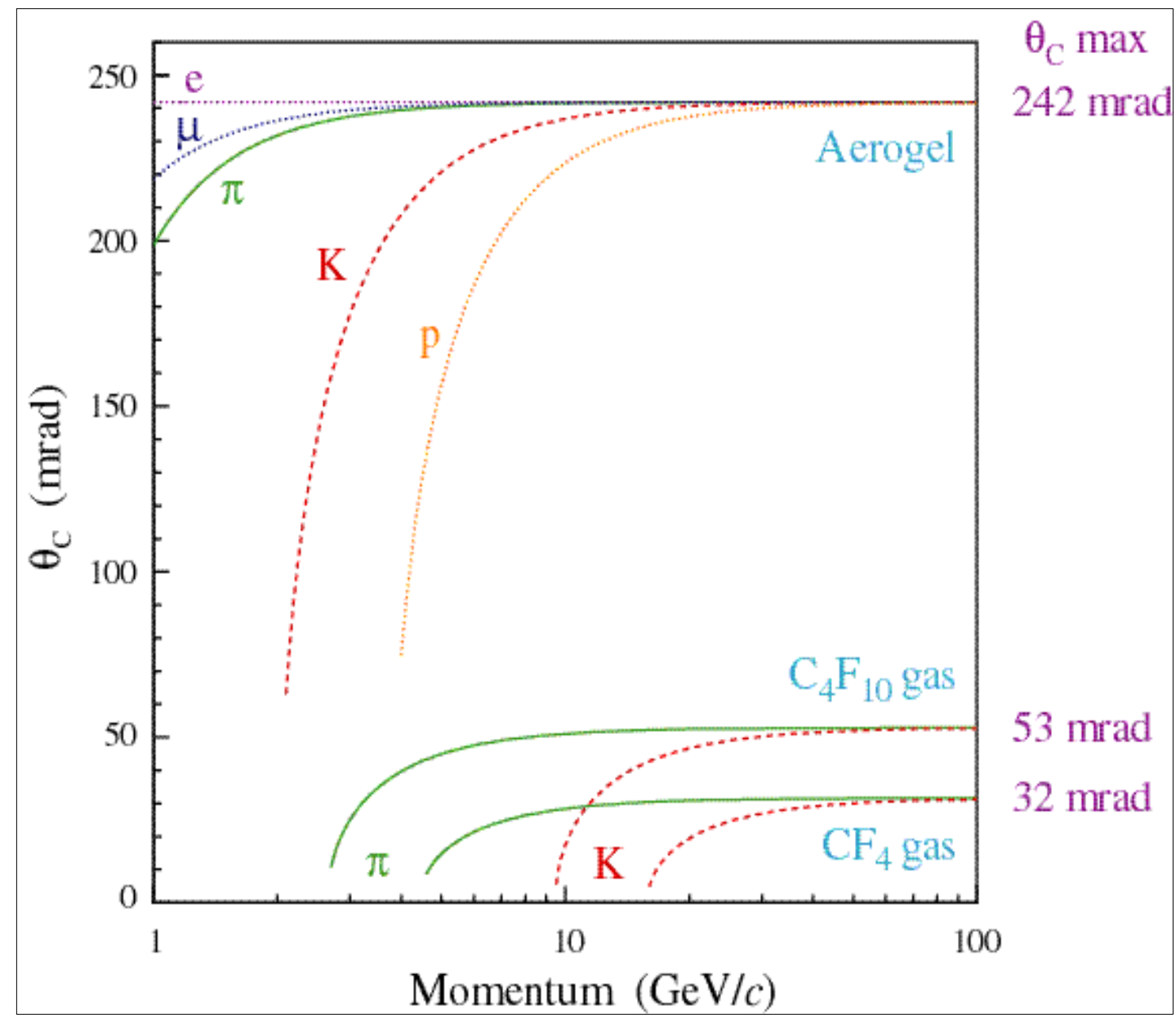




\section{Pixels' thresholds distribution}

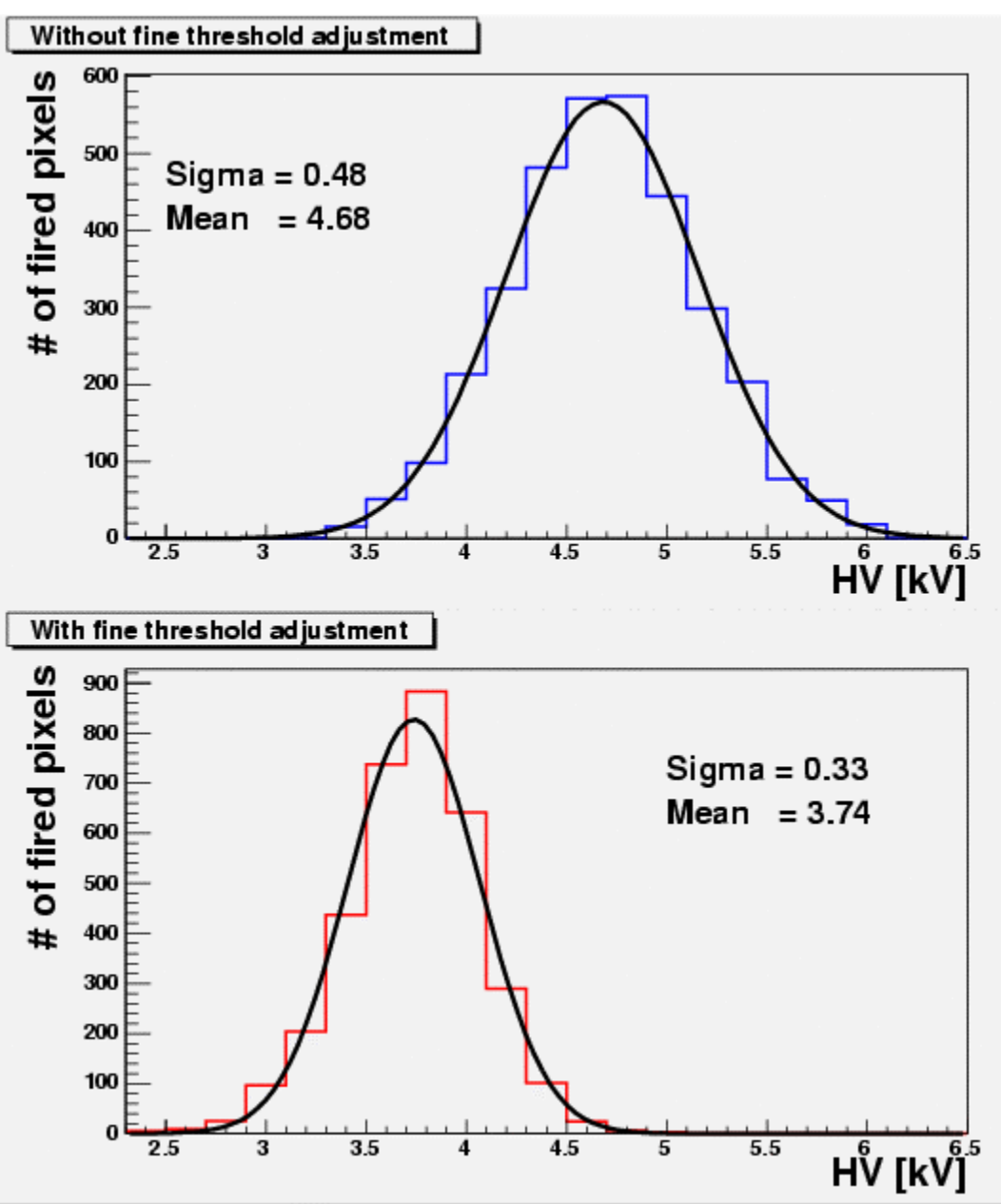

- Measurements with pulsed LED source in the laboratory

- Threshold distribution:

- avg: $4.68 \mathrm{kV} \sim 1300 \mathrm{e}$ (spec. < 2000e)

-rms: $0.48 \mathrm{kV} \sim 134 \mathrm{e}$ (spec. < 300e)

- 3-bit individual threshold adjustment allows for average threshold reduction and $1 \%$ improvement of detection efficiency at operational HV

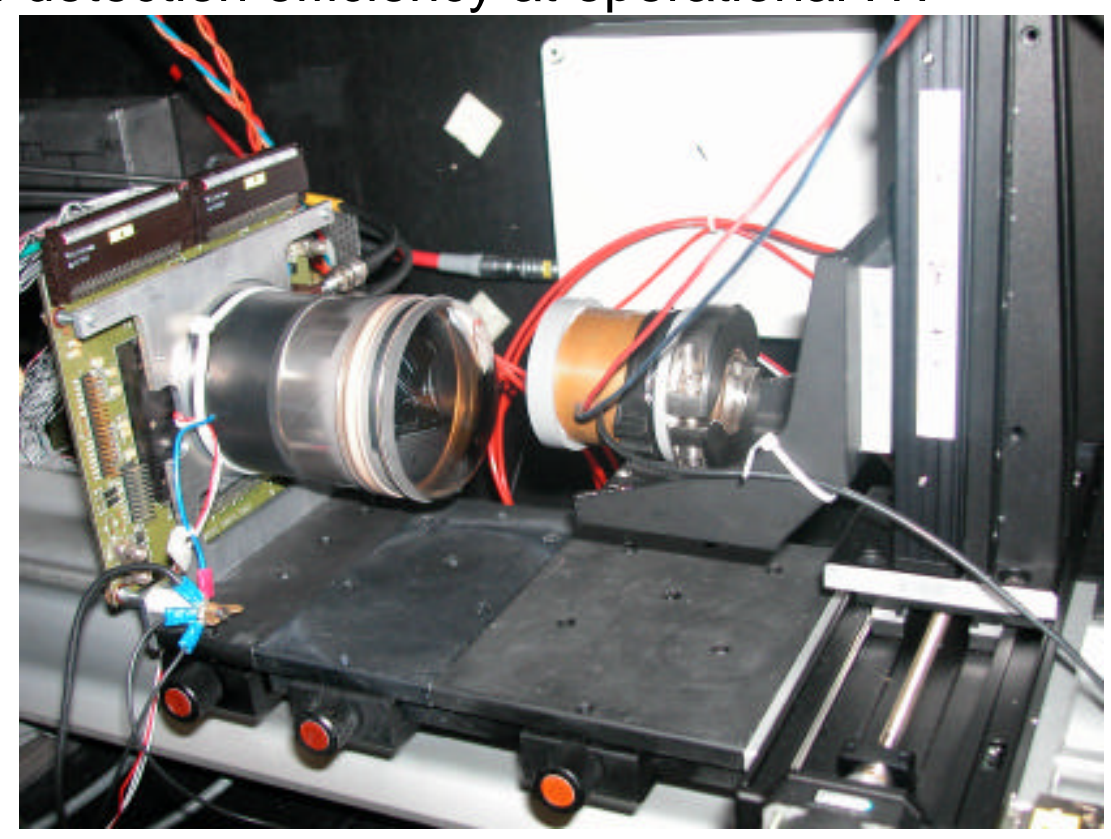

